\title{
Calidad de vida en personas con discapacidad física que practican actividad física
}

\author{
Quality of life in people with physical disabilities who perform physical activities \\ Kevin Jonathan Escajadillo Davalos 1,a, Melanie Francesca León Andonayre 1,a, \\ José Luis Rojas-Vilca ${ }^{1, b}$, María Luisa Sarmiento Almidón ${ }^{1, c}$
}

\section{RESUMEN}

Objetivo: Determinar la calidad de vida en personas con discapacidad física que realizan actividad física. Material y método: Estudio observacional, descriptivo y de corte transversal, que incluyó a deportistas con discapacidad física y utilizó el cuestionario SF-36 para medir la calidad de vida. Resultados: Se entrevistó a 60 personas, de las cuales el $86.7 \%$ mantenía una alta calidad de vida, en la que predominaron las dimensiones de vitalidad (98,3\%), función social $(83,3 \%)$ y dolor corporal (80,0\%); a diferencia de bienestar y salud (68.3\%) y función física (50.0\%) que contaron con una menor frecuencia de alta calidad de vida. No se halló diferencia estadísticamente significativa según edad, género ni causa de discapacidad física, pero sí el antecedente de haber participado en una competencia internacional, en aquellos que presentaron una menor calidad de vida. Conclusiones: La calidad de vida de los deportistas con discapacidad es alta, según la escala SF-36, mientras que es menor en el grupo con antecedente de participación en una competencia de alto nivel. Se recomienda realizar estudios que evalúen la influencia del deporte de alta competencia en la calidad de vida de deportistas con discapacidad física.

PALABRAS CLAVE: Discapacidad física, calidad de vida, actividad física.

\section{SUMMARY}

Objective: To determine the quality of life in people with physical disabilities who perform physical activities. Material and method: Observational, descriptive and cross-sectional study, which included athletes with physical disabilities, and used the SF-36 survey to measure their quality of life. Results: 60 people were interviewed, 86.7\% of them have a high quality of life with a predominance of the following dimensions: vitality (98.3\%), social function $(83.3 \%)$ and body pain $(80.0 \%)$, unlike the dimensions of health and welfare $(68.3 \%)$ and physical function $(50.0 \%)$, which were the ones with the lowest frequency in high quality of life. No statistically significant difference was found according to age, gender or cause of physical disability, but there is a history of participation in an international competition, in those that presented a lower quality of life. Conclusions: The quality of life of athletes with disabilities is high according to the SF-36 scale, but it is lower in the group with a history of participation in a high level competition. Future studies should evaluate the influence of highly competitive sports on the quality of life of athletes with physical disabilities.

KEYWORDS: physical disability, quality of life, physical activity.

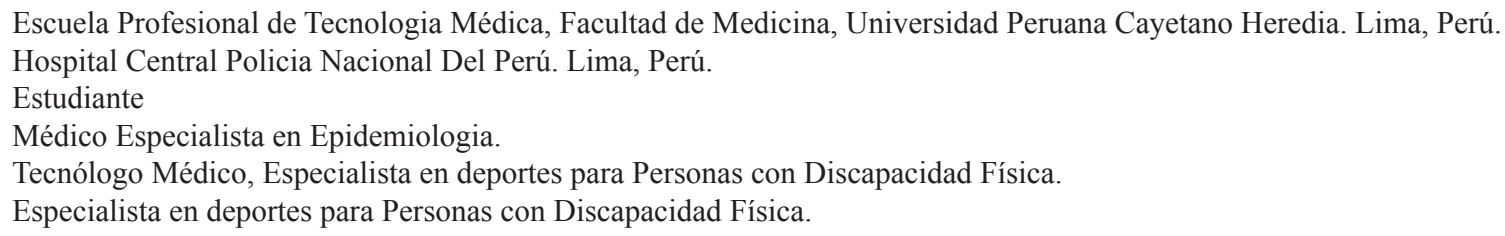




\section{INTRODUCCIÓN}

A partir de los últimos años del siglo XX se empezó a tomar mayor importancia al estudio sobre calidad de vida $(1,2)$. La Organización Mundial de la Salud (OMS) la define como "la percepción del individuo sobre su posición en la vida dentro del contexto cultural y el sistema de valores en el que vive y con respecto a sus metas, expectativas, normas y preocupaciones"(3); la definición ha demostrado tener capacidad predictiva tanto para la utilización de los servicios de salud como para morbimortalidad en diferentes enfermedades. En tal sentido, la calidad de vida se constituye como un indicador reconocido internacionalmente, de resultado o de necesidad en salud.

Otros autores, definen "calidad de vida" como las condiciones de vida que lleva todo individuo, dentro de las satisfacciones que experimenta con la educación que recibe y con el empleo; así como la apreciación que le otorgue a cada situación vivida, influenciada por el bienestar físico, mental y social, además del lugar donde reside. Todo esto dependerá de la cultura, ambiente, época y grupos sociales en los cuales la persona realice sus actividades. Por tanto, se considera muy importante como parte de la evaluación dentro de la salud de las personas, ya que influye en este aspecto, y se interviene en la parte de prevención, porque al tener una buena calidad de vida, se mejora y controla el estado de salud de las personas $(1,4,5)$.

Para la OMS, la discapacidad abarca las deficiencias, que afectan a la función corporal; las limitaciones al realizar tareas y las restricciones de la participación en situaciones de su día a día. En el 2001, la OMS aprobó la Clasificación Internacional del Funcionamiento, la Discapacidad y la Salud (CIF), para incluir el funcionamiento humano y la discapacidad como componentes de la salud; y establecer un lenguaje común entre los distintos profesionales que tienen contacto con personas que presentan algún tipo de discapacidad. Este modelo permite asociar aspectos desde las dimensiones corporal, individual y social, con los factores ambientales que pueden actuar como facilitadores o barreras de la situación de discapacidad (6-9).

Asimismo, cabe señalar que una persona presenta discapacidad cuando experimenta dificultades para poder realizar actividades que son necesarias para llevar una vida independiente, tanto en las actividades de la vida diaria (AVD) como en las actividades instrumentales de la vida diaria (AIVD). Las AVD son esenciales para el autocuidado de la persona; mientras que las AIVD requieren de mayor control y funcionamiento autónomo en un determinado entorno (9).

En el 2012, un total de 931993 personas a nivel nacional declararon, en la Primera Encuesta Nacional Especializada sobre Discapacidad, tener al menos una limitación física (3.1\% de la población total); de las cuales el $54,4 \%$ eran mujeres y el $31,5 \%$ se encontraba entre los 30 y 64 años(10). El 10,2\% de las personas que presentaban algún tipo de discapacidad física, tenían una lesión a consecuencia de ello, lo que se constituye en un factor de riesgo; y algunas de ellas sufrían de exclusión social(11).

Por otro lado, la actividad física se define como el movimiento corporal producido por la contracción de músculos esqueléticos que aumentan el gasto de energía por encima del nivel basal, aceptada a nivel mundial como una prioridad para poder prevenir enfermedades de tipo cardiovascular, obesidad y depresión. También mejora la capacidad músculo esquelética, la cual se asocia positivamente a la independencia funcional. Por tanto, una persona físicamente activa mejora su fuerza muscular, equilibrio y coordinación(12).

El tiempo recomendado para tener buenos resultados con la práctica de actividad física es de mínimo 5 días a la semana por 30 minutos diarios; estos tiempos pueden variar de acuerdo a las capacidades de cada persona(13-15).

Las personas con discapacidad pueden realizar cualquier actividad física adaptada, que si bien, antes solo se practicaba como medio de rehabilitación para contrarrestar la discapacidad física; en la actualidad es recomendada para aumentar el contacto social, mejorar la autoestima y generar una mejor imagen corporal de sí mismos(15-17,18). Sin embargo, es importante llevar a cabo una evaluación médica antes de comenzar a realizar algún tipo de actividad física, sea competitiva o no(19).

La tasa de participación en deportes y actividades físicas adaptadas es variable, de $11 \%$ a $60 \%$ en Estados Unidos y de 32\% a 39\% en los Países Bajos. Asimismo, notamos que es influenciada por factores como el nivel socioeconómico, la educación, cantidad de tiempo libre, edad y estado de salud(18).

Se espera que así como la actividad física mejora la calidad de vida en la población general $(16,20)$, 
también influya favorablemente en las personas con discapacidad, a pesar de los factores físicos que pudieran afectarla.

Este estudio tuvo por objetivo conocer el nivel de calidad de vida en deportistas con discapacidad física. El objetivo fue determinar la calidad de vida de personas con discapacidad física que practican actividad física, determinar la calidad de vida de personas con discapacidad física que practican actividad física, según género, edad y causa de la discapacidad física, y la calidad de vida de personas con discapacidad física, según su participación en alguna competencia internacional o no.

\section{MATERIAL Y MÉTODOS}

Se realizó un estudio de corte transversal, o encuesta entre los meses de setiembre del 2015 y enero del 2016.

La población de estudio estuvo conformada por todos los deportistas con alguna discapacidad física, mayores de 18 años, que aceptaron voluntariamente participar en la investigación. Se invitó a los afiliados a sedes de la Federación Deportiva Nacional de Personas con Discapacidad Física (FEDENADIF) en Lima y Callao: Asociación de Discapacitados de San Martín de Porres (ADISAMP), Leones de Carmen de la Legua y El sentir de la alegría. También fueron invitados los participantes de una competencia oficial nacional de paranatación realizada en la ciudad de Lima en el periodo de estudio, a la cual asistieron representantes de Arequipa, Piura, Lambayeque y Lima.

Para la investigación se utilizó el cuestionario de salud SF-36 validado en el Perú(21), el cual cuenta con 8 dimensiones y 36 preguntas; las 10 primeras son referidas a datos generales, mientras que las siguientes evalúan la percepción de la calidad de vida de la persona. Además, se elaboró una ficha de recolección de datos sobre características generales y antecedentes de los participantes (anexo 1).

Para la recolección de información se realizaron entrevistas individuales momentos previos a los entrenamientos, las cuales tuvieron una duración de 20 a 30 minutos. Para ello, se les informó del estudio, se solicitó su consentimiento informado y se les trasladó a un lugar más privado (anexo 2 ).
En el caso de los deportistas que se encontraban en la competencia nacional de para natación, las encuestas se realizaron de manera posterior a su participación, en un espacio más privado y previamente informados.

\section{RESULTADOS}

Se invitó a participar en el estudio a un total de 62 deportistas con discapacidad física, que cumplían con los criterios de selección, de los cuales 2 desistieron, quedando un total de 60 integrantes.

El $73,3 \%$ de los participantes eran de sexo masculino, con edad promedio de 41.0 años $(\mathrm{DE}= \pm$

Tabla 1. Análisis descriptivo de las variables sociodemográficas de los participantes.

\begin{tabular}{|c|c|}
\hline Variable sociodemográfica & n (\%) \\
\hline $\begin{array}{l}\text { Género } \\
\text { Femenino }\end{array}$ & $16(26,7)$ \\
\hline Masculino & $44(73.3)$ \\
\hline $\begin{array}{l}\text { Edad } \\
\text { Menor a } 20\end{array}$ & $3(5.0)$ \\
\hline 20 a 29 & $6(10.0)$ \\
\hline 30 a 39 & $15(25.0)$ \\
\hline 40 a 49 & $22(36.7)$ \\
\hline Mayor a 49 & $14(23.3)$ \\
\hline $\begin{array}{l}\text { Grado de instrucción } \\
\text { Primaria }\end{array}$ & $3(5.0)$ \\
\hline Secundaria & $36(60.0)$ \\
\hline Superior universitario & $21(35.0)$ \\
\hline $\begin{array}{l}\text { Ocupación } \\
\text { Jubilado }\end{array}$ & $1(1.7)$ \\
\hline Estudiante & $7(11.7)$ \\
\hline Ama de casa & $5(8.3)$ \\
\hline Empleado & $24(40.0)$ \\
\hline Independiente & $23(38.3)$ \\
\hline $\begin{array}{l}\text { Lugar de residencia } \\
\text { Lima norte }\end{array}$ & $17(28.3)$ \\
\hline Lima sur & $11(18.3)$ \\
\hline Lima centro & $10(16.7)$ \\
\hline Lima este & $4(6.7)$ \\
\hline Provincia Constitucional del Callao & $11(18.3)$ \\
\hline Interior del país & $7(11.7)$ \\
\hline $\begin{array}{l}\text { Sede } \\
\text { Carmen de la Legua y Reynoso }\end{array}$ & $13(21.7)$ \\
\hline San Borja & $19(31.7)$ \\
\hline San Martín de Porres & $18(30.0)$ \\
\hline Provincia & $10(16.6)$ \\
\hline
\end{tabular}


Tabla 2. Antecedentes de discapacidad física.

\begin{tabular}{clc}
\hline Variables & \multicolumn{1}{c}{ Categorías } & n (\%) \\
\hline & Secuela de polio & $37(61,6)$ \\
$\begin{array}{c}\text { Causa de } \\
\text { discapacidad }\end{array}$ & Lraumatismo & $3(5.0)$ \\
& $\begin{array}{l}\text { Lesión medular } \\
\text { central sistema nervioso }\end{array}$ & $12(20.0)$ \\
& Otros & $4(6.7)$ \\
& Superior derecho & $1(6.7)$ \\
& Inferior derecho & $8(13.3)$ \\
Miembro & Inferior izquierdo & $7(11.6)$ \\
afectado & Ambos miembros & $43(71.7)$ \\
& $\begin{array}{l}\text { inferiores } \\
\text { Ambos miembros }\end{array}$ & $1(1.7)$ \\
\hline
\end{tabular}

Tabla 3. Participación o no en alguna competencia internacional.

\begin{tabular}{lc}
\hline $\begin{array}{l}\text { Competencia } \\
\text { internacional }\end{array}$ & $\begin{array}{c}\text { Población } \\
\mathbf{n}(\%)\end{array}$ \\
\hline Sí participa & $25(41.7)$ \\
No participa & $35(58.3)$ \\
\hline
\end{tabular}

10,2), valor mínimo de 19 y máximo de 59 años. El $95 \%$ de la población estudiada tenía grado de instrucción secundaria o superior y el $78.3 \%$ tenía alguna actividad laboral al momento de realizar la investigación. El $83.4 \%$ de los participantes eran afiliados a la FEDENADIF de Lima y Callao (tabla 1).

En la población estudiada la principal causa de discapacidad fue la secuela de polio $(61.6 \%)$, los miembros más afectados fueron ambas extremidades inferiores $(71,7 \%$ ) (tabla 2$)$; y 25 de ellos $(41,7 \%)$ tuvieron como antecedente el haber participado en alguna competencia internacional (tabla 3 ).

Según el cuestionario SF-36, 52 de los participantes $(86,7 \%)$ tuvieron alta calidad de vida global $(>50$ puntos); el promedio del puntaje de todas las dimensiones de estudio evaluadas estuvo por encima del punto de cohorte $(>50)$ indicando buena calidad de vida; sin embargo, se observó una mayor frecuencia de bajo nivel de calidad de vida en las dimensiones bienestar y salud $(68,3 \%)$ así como en función física $(50,0 \%)$ (tabla 4$)$.

La calidad de vida no presentó diferencias estadísticamente significativas según género, edad ni causa de discapacidad física (tabla 5). Al evaluar la calidad de vida de los deportistas, según su participación o no en alguna competencia internacional, esta resultó

Tabla 4. Calidad de Vida y dimensiones en deportistas con discapacidad física.

\begin{tabular}{lrrr}
\multicolumn{1}{c}{ Dimensiones } & $\begin{array}{r}\text { Baja } \\
\mathbf{n}(\mathbf{\%})\end{array}$ & $\begin{array}{r}\text { Alta } \\
\mathbf{n}(\mathbf{\%})\end{array}$ & Promedio Puntaje \\
\hline Calidad de Vida & $8(13.3)$ & $52(86.7)$ & 71.6 \\
$\quad$ Bienestar y salud & $41(68.3)$ & $19(31.7)$ & 57.5 \\
Evolución de la salud & $28(46.7)$ & $32(53.3)$ & 89.8 \\
Salud general & $18(30.0)$ & $42(70.0)$ & 87.3 \\
Función física & $30(50.0)$ & $30(50.0)$ & 72.7 \\
Rol físico & $19(31.7)$ & $41(68.3)$ & 93.3 \\
Salud mental & $15(25.0)$ & $45(75.0)$ & 97.0 \\
Función social & $10(16.7)$ & $50(83.3)$ & 80.0 \\
Dolor corporal & $12(20.0)$ & $48(80.0)$ & 75.3 \\
Vitalidad & $1(1.7)$ & $59(98.3)$ & 76.6 \\
\hline
\end{tabular}


Tabla 5. Calidad de vida según género, edad y causa de discapacidad física

\begin{tabular}{|c|c|c|c|}
\hline Variable & $\begin{array}{c}\text { Alta calidad de vida } \\
\text { n (\%) }\end{array}$ & $\begin{array}{c}\text { Baja calidad de vida } \\
\text { n (\%) }\end{array}$ & Chi2 (p) \\
\hline $\begin{array}{l}\text { Género } \\
\text { Femenino }\end{array}$ & $14(87.5)$ & $2(125)$ & \\
\hline Masculino & $38(86.4)$ & $\begin{array}{l}2(12.5) \\
6(13.6)\end{array}$ & $\begin{array}{l}0.0131 \\
(0.909)\end{array}$ \\
\hline $\begin{array}{l}\text { Edad } \\
\text { Menor a } 20\end{array}$ & $3(100.0)$ & $0(0.0)$ & \multirow{5}{*}{$\begin{array}{l}3.6976 \\
(0.448)\end{array}$} \\
\hline 20 a 29 & $5(83.3)$ & $1(16.7)$ & \\
\hline 30 a 39 & $12(80.0)$ & $3(20.0)$ & \\
\hline 40 a 49 & $18(81.8)$ & $4(18.2)$ & \\
\hline Mayor a 49 & $14(100.0)$ & $0(0.0)$ & \\
\hline $\begin{array}{l}\text { Causa de discapacidad } \\
\text { Secuela de polio }\end{array}$ & $31(83.7)$ & $6(16.3)$ & \multirow{5}{*}{$\begin{array}{l}2.0738 \\
(0.722)\end{array}$} \\
\hline Traumatismo & $3(100.0)$ & $0(0.0)$ & \\
\hline Lesión medular & $11(91.7)$ & $1(8.3)$ & \\
\hline $\begin{array}{l}\text { Lesión sistema } \\
\text { nervioso central }\end{array}$ & $3(75.0)$ & $1(25.0)$ & \\
\hline Otros & $4(100.0)$ & $0(0.0)$ & \\
\hline
\end{tabular}

Tabla 6. Puntaje de calidad de vida global y por dimensiones (SF-36) en deportistas con discapacidad física, según participación en competencia internacional

\begin{tabular}{lcccc}
\hline \multirow{2}{*}{ Calidad de vida } & \multicolumn{2}{c}{ Competencia internacional } & & \\
\cline { 2 - 3 } & $\begin{array}{c}\text { Sí } \\
\mathbf{x}(\mathbf{D E})\end{array}$ & $\begin{array}{c}\mathbf{N o} \\
\mathbf{x}(\mathbf{D E})\end{array}$ & & p \\
\hline Calidad de vida & $62.9( \pm 15.6)$ & $70.9( \pm 11.7)$ & -2.177 & $* 0.035$ \\
$\quad$ Bienestar y salud & $66.1( \pm 20.3)$ & $73.9( \pm 18.3)$ & -1.526 & 0.134 \\
Evolución de la salud & $62.0( \pm 28.1)$ & $72.1( \pm 25.6)$ & -1.432 & 0.159 \\
Salud general & $65.8( \pm 28.1)$ & $75.9( \pm 25.3)$ & 1.427 & 0.159 \\
Función física & $53.6( \pm 26.4)$ & $51.1( \pm 22.8)$ & 0.376 & 0.708 \\
Rol físico & $58.0( \pm 36.6)$ & $79.3( \pm 35.6)$ & -2.246 & $* 0.029$ \\
Salud mental & $58.7( \pm 42.2)$ & $88.6( \pm 30.2)$ & -3.026 & $* 0.004$ \\
Función social & $70.0( \pm 17.3)$ & $76.4( \pm 20.5)$ & -1.312 & 0.195 \\
Dolor corporal & $59.9( \pm 22.3)$ & $72.5( \pm 21.9)$ & -2.175 & $* 0.034$ \\
Vitalidad & $74.2( \pm 13.5)$ & $77.3( \pm 12.7)$ & -0.881 & 0.382 \\
\hline
\end{tabular}

Nota: $\mathrm{p}$-valor $<0.05$ "Significativo".

mayor en quienes no la tuvieron (prueba $\mathrm{t}=-2,177$; valor $\mathrm{p}<0,05$ ). Se obtuvo también una diferencia significativa en las dimensiones de rol físico, salud corporal y dolor corporal (tabla 6).

\section{DISCUSIÓN}

La mayor frecuencia de alta calidad de vida encontrada en los deportistas con discapacidad física $(86,7 \%)$ es cercana a lo encontrado por Paredes $(94,1 \%)$ 
en personas sin discapacidad física que asisten a un gimnasio, y se corresponde con las investigaciones de Henschen y Horvat sobre jugadores de baloncesto en silla de ruedas, quienes refieren sentirse más seguros de sí mismos y con mayor autocontrol; la motivación y el sentirse más competentes influyen favorablemente en la percepción de su salud(22-26).

Algunos autores señalan que la única diferencia entre los jugadores con discapacidad física y los que no la tienen, es la mayor fatiga luego de una competencia debido a la mayor demanda de energía $(27,28)$. Diversos estudios señalan que la actividad física y el deporte están asociados a una mayor calidad de vida, por lo que se recomienda realizar alguna actividad física $(16,20,29,30)$.

Las 3 dimensiones con mayor calidad de vida en este estudio (vitalidad, función social y dolor corporal) concuerdan con otras investigaciones, cuyos resultados afirman que las personas con discapacidad que realizan actividades físicas observan mejoras en su desarrollo emocional(31) y social(31,32), favorecidas por las relaciones sociales establecidas en entrenamientos y competiciones, donde se potencian valores como el trabajo en equipo y se refuerzan su autoconcepto y autoestima.

Si bien la calidad de vida global y salud, así como la percepción de bienestar psicológico aumentan junto con el nivel de competencia deportiva(32), en nuestra población estas dimensiones estarían directamente afectadas por la discapacidad física, por lo cual no alcanzan valores tan altos como los mencionados.

La vitalidad se asocia directamente con el nivel de energía, entusiasmo, la actividad que se realiza y el lugar en el cual se desarrolla, así como el clima motivacional y ego de la persona. En ese sentido, la actividad física puede influir en ello, ya que al canalizar situaciones de estrés, ansiedad y preocupaciones se ayuda a mejorar las capacidades del individuo, lo que a su vez incrementa sus competencias y destrezas personales(33-35).

Aunque, se esperaría que quienes participan en alguna competencia internacional tengan una mayor capacidad funcional en las AVD (dimensión rol físico), nuestros resultados demuestran lo contrario; esto podría explicarse por el mayor riesgo de sufrir lesiones osteomioarticulares(28), lo cual fue evidenciado en deportistas de baloncesto sin discapacidad física por Lozana(36).
De la misma manera, la dimensión de "salud mental" denota una menor frecuencia de alta calidad de vida en quienes participaron en alguna competencia internacional. A pesar de que algunos estudios demuestran que a mayor exigencia se disminuiría los índices de depresión y melancolía(37); otros refieren que la ansiedad es estable luego de participar en estos eventos y se eleva de dos a cuatro días antes de una competencia(38), aunque también podría elevarse por el estrés a sufrir lesiones(39).

En conclusión, el nivel de calidad de vida de los deportistas con discapacidad física evaluados es alta, sin mostrar diferencia por género, edad o causa de discapacidad física. Sin embargo, cuando el deportista discapacitado ha participado en alguna competencia internacional, la calidad de vida es menor en comparación con aquellos que no lo hicieron.

Lo anterior, nos hace pensar si se trata de una condición propia de la evolución de su discapacidad física $\mathrm{y} / \mathrm{u}$ otra patología, o podría estar relacionado a la experiencia de lesiones debido a la mayor demanda de esfuerzo físico(27,28). Ante esto, algunos estudios recomiendan la intervención del profesional en fisioterapia para la prevención de lesiones osteomioarticulares(39), así como el apoyo de un equipo multidisciplinario.

Por otra parte, cabe destacar que son limitaciones del estudio la selección por conveniencia y el reducido tamaño de la población, así también la falta de información sobre antecedentes de lesiones osteomioarticulares y la proximidad a una competencia oficial, para poder evaluar si estos factores podrían estar relacionados con la calidad de vida.

\section{Correspondencia}

José Luis Rojas-Vilca

Correo electrónico: jose.rojas@upch.pe

\section{REFERENCIAS BIBLIOGRAFICAS}

1. Ardila R. Calidad de vida: una definición integradora. Rev Latinoam Psicol. 2003;35(2):161-164. (Citado 30 de agosto de 2017) Disponible en: http://www. redalyc.org/pdf/805/80535203.pdf

2. Gómez M, Sabeh E. Calidad de vida. Evolución del concepto y su influencia en la investigación y la práctica. Salamanca: Fac Psicol, Universidad Salamanca; 2001. (Citado 30 de agosto de

2017) Disponible en: http://www.academia.edu/ download/38683739/CALIDAD_DE_VIDA.pdf

3. Group W. The World Health Organization quality 
of life assessment (WHOQOL): position paper from the World Health Organization. Soc Sci Med. 1995;41(10):1403-1409.

4. Velarde-Jurado E, Avila-Figueroa C. Evaluación de la calidaddevida.SaludPúblicaMéxico.2002;44(4):349361. (Citado 30 de agosto de 2017) Disponible en: http://www.scielo.org.mx/scielo.php?script=sci_artte xt\&pid=S0036-36342002000400009

5. Awuapara N. Determinación de la asociación entre el edentulismo y la calidad de vida en la población adulta del distrito de Celendín, provincia de Celendín, departamento de Cajamarca en 2010. Lima Perú: Univ Peru Cayetano Heredia. 2010;

6. World Health Organization. International classification of functioning, disability and health: ICF. Washington DC: World Health Organization; 2001.

7. Zeldenryk L, Gordon S, Gray M, Speare R, Melrose W. Disability measurement for lymphatic filariasis: a review of generic tools used within morbidity management programs. PLoS Negl Trop Dis. 2012;6(9):e1768. (Citado 30 de agosto de 2017) Disponible en: http://journals.plos.org/plosntds/ article?id=10.1371/journal.pntd.0001768

8. Fernández-López JA, Fernández-Fidalgo M, Geoffrey R, Stucki G, Cieza A. Funcionamiento y discapacidad: la clasificación internacional del funcionamiento (CIF). Rev Esp Salud Pública. 2009 ;83(6):775783. (Citado 30 de agosto de 2017) Disponible en: http://scielo.isciii.es/scielo.php?script=sci_arttext\&p $\mathrm{id}=$ s1135-57272009000600002

9. González S, Tello J, Silva P, Lüders C, Butelmann $\mathrm{S}$, Fristch R, et al. Calidad de vida en pacientes con discapacidad motora según factores sociodemográficos y salud mental. Rev Chil NeuroPsiquiatr. 2012;50(1):23-34. (Citado 30 de agosto de 2017) Disponible en: http://www.scielo.cl/scielo. php?pid=S0717-92272012000100003\&script $=$ sci arttext\&tlng=en

10. Instituto Nacional de Estadística e Informática. Encuestas. Lima: Instituto Nacional de Estadística e Informática;2017.

11. Zhu H, Xia X, Xiang H, Yu C, Du Y. Disability, home physical environment and non-fatal injuries among young children in China. PloS One. 2012;7(5):e37766. (Citado 30 de agosto de 2017) Disponible en: http:// journals.plos.org/plosone/article?id=10.1371/ journal.pone.0037766

12. Luctkar-Flude M. Fatigue, physical activity, physical functioning and quality of life in older adults with cancer. Ottawa: University of Ottawa; 2007. (Citado 30 de agosto de 2017) Disponible en: http://www. ruor.uottawa.ca/handle/10393/27883

13. Shirley D, Van der Ploeg HP, Bauman AE. Physical activity promotion in the physical therapy setting: perspectives from practitioners and students. Phys
Ther. 2016; 90(9):1311-1322. (Citado 30 de agosto de 2017) Disponible en: https://academic.oup.com/ ptj/article-abstract/90/9/1311/2738092

14. Wagenmakers R, Stevens M, Groothoff JW, et al. Physical activity behavior of patients 1 year after primary total hip arthroplasty: a prospective multicenter cohort study. Phys Ther. 2011;91(3):373380. (Citado 30 de agosto de 2017) Disponible en: https://academic.oup.com/ptj/article-abstract/ 91/3/373/2735015

15. Kersten RF, Stevens M, van Raay JJ, Bulstra SK, van den Akker-Scheek I. Habitual physical activity after total knee replacement. Phys Ther. 2012;92(9):1109-1116. (Citado 30 de agosto de 2017) Disponible en: https://academic.oup.com/ptj/ article-abstract/92/9/1109/2735270

16. Guillén F, Castro JJ, Guillén MÁ. Calidad de vida, salud y ejercicio físico. Rev Psicol Deporte. 1997;6(2):0091-110. (Citado 30 de agosto de 2017) Disponible en: https://dd.uab.cat/record/63598

17. Córdoba-Andrade L, Gómez-Benito J, VerdugoAlonso MÁ. Calidad de vida familiar en personas con discapacidad: un análisis comparativo. Univ Psychol; 2008; 7(2). (Citado 30 de agosto de 2017) Disponible en: http:/www.redalyc.org/html/647/64770206/

18. Bragaru M, van Wilgen CP, Geertzen JHB, Ruijs SGJB, Dijkstra PU, Dekker R. Barriers and Facilitators of Participation in Sports: A Qualitative Study on Dutch Individuals with Lower Limb Amputation. PLoS ONE. 2013; 8(3):e59881. (Citado 30 de agosto de 2017) Disponible en: http://dx.plos. org/10.1371/journal.pone.0059881

19. Gutiérrez M, Caus N. Análisis de los motivos para la participación en actividades físicas de personas con y sin discapacidad. Rev Int Cienc Deporte. 2006;2(2):49-64.

20. Cóndor JD. Calidad de vida y estado de salud de estudiantes que realizan actividades extracurriculares en la Pontificia Universidad Católica del Ecuador desde el contexto de la promoción de salud. Quito: PUCE; 2016. (Citado 30 de agosto de 2017) Disponible en: http://repositorio.puce.edu.ec/handle/22000/ 12826

21. Salazar FR, Bernabé E. The Spanish SF-36 in Peru: Factor Structure, Construct Validity, and Internal Consistency. Asia Pac J Public Health. 2015;27(2):NP2372-NP2380. (Citado 30 de agosto de 2017) Disponible en: http://journals.sagepub.com/ doi/10.1177/1010539511432879

22. Henschen KP, Horvat M, Roswal G. Psychological profiles of the United States wheelchair basketball team. Int J Sport Psychol. 1992. (Citado 30 de agosto de 2017) Disponible en: http://psycnet.apa. org/psycinfo/1993-18202-001

23. Horvat M, French R, Henschen K. A comparison of the psychological characteristics of male and 
female able-bodied and wheelchair athletes. Spinal Cord. $1986 ; 24(2): 115-122$. (Citado 30 de agosto de 2017) Disponible en: https://www.nature.com/sc/ journal/v24/n2/abs/sc198615a.html

24. Paredes RD. Relación de la actividad física con la calidad de vida en personas que realizan actividad física regular en el gimnasio Stampa en el Valle de los Chillos, en contraste con personas sedentarias de la empresa Farmacéutica James Brown en el período de octubre a diciembre del 2015. Tesis de Grado. Quito: PUCE; 2015. (Citado 30 de agosto de 2017) Disponible en: http://repositorio.puce.edu.ec/handle/22000/ 10434

25. Castillo I, Molina-García J, Álvarez O. Importancia de la percepción de competencia y de la motivación en la salud mental de deportistas universitarios. Salud Pública México. 2010;52(6):517-523. (Citado 30 de agosto de 2017) Disponible en: http:// www.scielo.org. $\mathrm{mx} / \mathrm{scielo} . \mathrm{php}$ ? pid=S0036$36342010000600006 \&$ script $=$ sci_arttext\&tlng $=\mathrm{pt}$

26. De la Vega R, Galán Á, Ruiz R, Tejero CM. Estado de ánimo precompetitivo y rendimiento percibido en Boccia Paralímpica. Rev Psicol Deporte. 2013;22(1). (Citado 30 de agosto de 2017) Disponible en: http:// www.redalyc.org/html/2351/235127552006/

27. Paulsen P, French R, Sherrill C. Comparison of mood states of college able-bodied and wheelchair basketball players. Percept Mot Skills. 1991;73(2):396398. (Citado 30 de agosto de 2017) Disponible en: http://journals.sagepub.com/doi/abs/10.2466/ pms.1991.73.2.396

28. Paulsen P, French R, Sherrill C. Comparison of wheelchair athletes and nonathletes on selected mood states. Percept Mot Skills. 1990;71(3 suppl):1160-1162. (Citado 30 de agosto de 2017) Disponible en: http://journals.sagepub.com/doi/ abs/10.2466/pms.1990.71.3f.1160

29. Ramírez W, Vinaccia S, Suárez GR. El impacto de la actividad física y el deporte sobre la salud, la cognición, la socialización y el rendimiento académico: una revisión teórica. Rev Estud Soc. 2004;(18):67-75. (Citado 30 de agosto de 2017) Disponible en: http://www.scielo.org.co/scielo. php?pid=S0123-885X2004000200008\&script $=$ sci arttext\&tlng=es

30. Caspersen CJ, Powell KE, Christenson GM. Physical activity, exercise, and physical fitness: definitions and distinctions for health-related research. Public Health Rep. 1985;100(2):126. (Citado 30 de agosto de 2017) Disponible en: https://www.ncbi.nlm.nih.gov/pmc/ articles/pmc1424733/

31. Macías D, González López I. Inclusión social de personas con discapacidad física a través de la natación de alto rendimiento. Apunts Educ Física
Deport. 2012;(110):26-35. (Citado 30 de agosto de 2017) Disponible en: http://www.revista-apunts.com/ es/hemeroteca? article $=1562$

32. Jürgens I. Práctica deportiva y percepción de calidad de vida. Rev Int Med Cienc Act Física Deport J Med Sci Phys Act Sport. 2006;6(22). (Citado 30 de agosto de 2017) Disponible en: http://www.redalyc. org $/ \mathrm{html} / 542 / 54221990001 /$

33. Moncayo Crespo HA. Documental: Reinterpretación del cuerpo a través del Tai Chi como expresión corporal y comunicativa para mejorar las condiciones de vitalidad y del buen vivir. Tesis: Quito: Universidad Politecnica Salesiana; 2015 (Citado 30 de agosto de 2017) Disponible en: http://dspace.ups. edu.ec/handle/123456789/9790

34. Balaguer I, Castillo I, Duda JL, García-Merita M. Asociaciones entre la percepción del clima motivacional creado por el entrenador, orientaciones disposicionales de meta, regulaciones motivacionales y vitalidad subjetiva en jóvenes jugadoras de tenis. Rev Psicol Deporte. 2011(Citado 30 de agosto de 2017) Disponible en: http://www.redalyc. org $/ \mathrm{html} / 2351 / 235119302010 /$

35. Molina-García J, Castillo I, Pablos C. Bienestar psicológico y práctica deportiva en universitarios.

Mot Eur J Hum Mov. 2007;18:79-91. (Citado 30 de agosto de 2017) Disponible en: http://www.redalyc. org/pdf/2742/274220374005.pdf

36. Lozana CB, Pereira JS. Frequência de lesões osteomioarticulares e tempo de afastamento das atividades esportivas em atletas de basquetebol de alto rendimento. Fit Perform J. 2003;2(1):17-22. (Citado 30 de agosto de 2017) Disponible en: http:// fpjournal.org.br/painel/arquivos/2035-2_Basquete_ Rev1_2003_Portugues.pdf

37. Segura J, Molist E, Arcarons M, Piqué N. Práctica deportiva y estado de ánimo en deportistas discapacitados físicos. Apunts Med Esport. 1999;34(131):11-18. (Citado 30 de agosto de 2017) Disponible en: http://www.sciencedirect.com/ science/article/pii/S1886658199759335

38. Ries F, Castañeda C, Campos M del C, Andrés C, Del O. Relaciones entre ansiedad-rasgo y ansiedadestado en competiciones deportivas. Cuad Psicol Deporte. 2012;12(2):9-16. (Citado 30 de agosto de 2017) Disponible en: http://scielo.isciii.es/scielo. php?pid=S1578-84232012000200002\&script $=$ sci arttext\&tlng=en

39. García O, Cancela JM, Olveira E, Mariño R. ¿Es compatible el máximo rendimiento deportivo con la consecución y mantenimiento de un estado saludable del deportista? RICYDE. 2009;(14). (Citado 30 de agosto de 2017) Disponible en: http:// www.redalyc.org/html/710/71011547004/ 
Anexo 1. Fichas de recolección

Ficha de recolección de datos generales

Código:

Distrito:

Ocupación:

Nivel de discapacidad:

\begin{tabular}{|l|l|l|}
\hline & Derecha & Izquierda \\
\hline Miembro superior & & \\
\hline Miembro inferior & & \\
\hline
\end{tabular}

Causas de discapacidad física:

\begin{tabular}{|l|l|l|l|}
\hline Lesión medular & Secuela de Polio & \\
\hline Traumatológica & & Lesión del Sistema Nervioso Central & \\
\hline Pie Diabético & Otros & \\
\hline
\end{tabular}

\begin{tabular}{|l|l|l|l|l|l|}
\hline Carmen de la Legua & & San Borja & & San Martin de Porres & \\
\hline
\end{tabular}

Sede de FEDENADIF:

¿Qué deporte practica?

¿Representó al Perú en alguna competencia internacional?

\begin{tabular}{|l|l|}
\hline SI & \\
\hline NO & \\
\hline
\end{tabular}

Si su respuesta es sí, ¿en qué deporte? 
Anexo 2. Cuestionario sf-36 sobre el estado de salud

\section{Instrucciones:}

Las preguntas que siguen se refieren a lo que usted piensa sobre su salud. Sus respuestas permitirán saber cómo se encuentra usted y hasta qué punto es capaz de hacer sus actividades habituales.

Conteste cada pregunta tal como se indica. Si no está seguro/a de cómo responder a una pregunta, por favor conteste lo que le parezca más cierto.

\section{DATOS GENERALES DEL ENCUESTADO.}

Sexo:

Femenino....................

Masculino...................1

Edad en años cumplidos: Años

Grado de instrucción:

Analfabeto....................... 0

Primaria........................... 1

Secundaria........................2

Superior universitario.............3

Diagnostico principal:

Diabetes mellitus................1

Cáncer............................2

Hipertensión.........................3

Tuberculosis....................4

SIDA ..........................

\section{¿Cuál es el material predominante en las paredes externas de su vivienda?}

Ladrillo o bloque de cemento..........1 Piedra con barro...................5

Piedra o sillas con cal o cemento......2 Madera...........................6

Adobe o tapia.......................... $\quad$ Estera................................

Quincha (caña con barro).............4 Otro material....................8

La vivienda tiene servicio higiénico (wáter, excusado o letrina), conectado a:

Red pública de desagüe...................1 Rio, acequia, canal o similares........

Pozo ciego o negro/séptico................2 No tiene servicio higiénico............

Sin contar baño, cocina, pasadizos ni garaje ¿Cuántas habitaciones, en total tiene su vivienda?

Número total de habitaciones.

En su hogar ¿tiene alguno de los siguiente servicios? (puede marcar más de una alternativa)

\begin{tabular}{|ll|}
\hline Internet.................... & Teléfono celular..................4 \\
TV por cable............... & Ninguno de los anteriores........... \\
Teléfono fijo.............. 3 & \\
\hline
\end{tabular}


En su hogar ¿tiene alguno de los siguientes artefactos? (puede marcar más de una alternativa)

\begin{tabular}{|c|c|}
\hline TV a color & Refrigeradora/congeladora.... \\
\hline Equipo de sonido... & Computadora................. \\
\hline Video grabadora $\ldots \ldots \ldots \ldots \ldots \ldots \ldots \ldots \ldots \ldots \ldots$ & Terma............... \\
\hline Horno microondas......................... 4 & Vehículo de uso particular............9 \\
\hline Lavadora.................... & Vehículo de trabajo........... \\
\hline
\end{tabular}

\section{CUESTIONARIO DE SALUD}

En general, usted diría que su salud es: (marque un solo número)

Excelente.........................1

Muy buena.....................2

Buena........................... 3

Regular........................4

Mala.............................5

¿Cómo diría usted que es su salud, comparada con la de hace un año? (marque un solo número)

Mucho mejor ahora que hace un año....1

Algo mejor ahora que hace un año.......2
Más o menos igual que hace un año........3

Algo peor ahora que hace un año........ 4

Mucho peor ahora que hace un año.......5

Las siguientes preguntas se refieren a actividades o cosas que usted podría hacer en un día normal. Su salud actual, ¿le limita para hacer esas actividades o cosas? Si es así, ¿Cuánto? (marque un solo número por cada pregunta)

\begin{tabular}{|l|c|c|c|}
\hline \multicolumn{1}{|c|}{ ACTIVIDADES } & $\begin{array}{l}\text { Sí, me limita } \\
\text { mucho }\end{array}$ & $\begin{array}{l}\text { Sí, me limita } \\
\text { un poco }\end{array}$ & $\begin{array}{l}\text { No, me limita } \\
\text { nada }\end{array}$ \\
\hline a. $\begin{array}{l}\text { Esfuerzos intensos, tales como correr, levantar objetos } \\
\text { pesado, o participar en deportes agotadores. }\end{array}$ & $\mathbf{1}$ & $\mathbf{2}$ & $\mathbf{3}$ \\
\hline $\begin{array}{l}\text { b. } \quad \begin{array}{l}\text { Esfuerzos moderados, como mover una mesa, pasar la } \\
\text { aspiradora, jugar a los bolos o caminar más de 1 hora. }\end{array} \\
\text { c. } \text { Coger o llevar la bolsa de compras. }\end{array}$ & $\mathbf{1}$ & $\mathbf{2}$ & $\mathbf{3}$ \\
\hline d. subir varios pisos por la escalera & $\mathbf{1}$ & $\mathbf{2}$ & $\mathbf{3}$ \\
\hline e. subir un solo piso por la escalera. & $\mathbf{1}$ & $\mathbf{2}$ & $\mathbf{3}$ \\
\hline f. Agacharse, arrodillarse o ponerse en cunclillas. & $\mathbf{1}$ & $\mathbf{2}$ & $\mathbf{3}$ \\
\hline g. Caminar un kilómetro o más. & $\mathbf{1}$ & $\mathbf{2}$ & $\mathbf{3}$ \\
\hline $\begin{array}{l}\text { h. } \text { Caminar varias manzanas (varios centenares de } \\
\text { metros). }\end{array}$ & $\mathbf{1}$ & $\mathbf{2}$ & $\mathbf{3}$ \\
\hline i. Caminar una sola manzana (unos 100 metros) & $\mathbf{1}$ & $\mathbf{2}$ & $\mathbf{3}$ \\
\hline j. Bañase o vestirse por sí mismo & $\mathbf{1}$ & $\mathbf{3}$ \\
\hline
\end{tabular}

Durante las 4 últimas semanas, ¿ha tenido alguno de los siguientes problemas en su trabajo o en sus actividades cotidianas, a causa su salud física? (marque un solo número por cada pregunta)

\begin{tabular}{|l|c|c|}
\hline & SI & NO \\
\hline a. ituvo que reducir el tiempo dedicado al trabajo o a sus actividades cotidianas? & $\mathbf{1}$ & $\mathbf{2}$ \\
\hline b. ¿hizo menos de lo que hubiera querido hacer? & $\mathbf{1}$ & $\mathbf{2}$ \\
\hline $\begin{array}{l}\text { c. ituvo que dejar de hacer algunas tareas en su trabajo o en sus actividades } \\
\text { cotidianas? }\end{array}$ & $\mathbf{1}$ & $\mathbf{2}$ \\
\hline $\begin{array}{l}\text { d. ituvo dificultad para hacer su trabajo o sus actividades cotidianas (por ejemplo, le } \\
\text { costó más de los normal)? }\end{array}$ & $\mathbf{1}$ & $\mathbf{2}$ \\
\hline
\end{tabular}


Durante las 4 últimas semanas, ¿ha tenido alguno de los siguientes problemas en su trabajo o en sus actividades cotidianas, a causa de algún problema emocional (como estar triste, deprimido, o nervioso)? (marque un solo número por cada pregunta)

\begin{tabular}{|c|c|c|}
\hline & SI & NO \\
\hline $\begin{array}{l}\text { a. ¿Tuvo que reducir el tiempo dedicado al trabajo o a sus actividades cotidianas, } \\
\text { por algún problema emocional? }\end{array}$ & $\mathbf{1}$ & $\mathbf{2}$ \\
\hline b. ¿Hizo menos de lo que hubiera querido hacer, por algún problema emocional? & 1 & 2 \\
\hline $\begin{array}{l}\text { c. ¿No hizo su trabajo o sus actividades cotidianas tan cuidadosamente como de } \\
\text { costumbre, por algún problema emocional? }\end{array}$ & $\mathbf{1}$ & $\mathbf{2}$ \\
\hline
\end{tabular}

Durante las 4 últimas semanas, ¿hasta qué punto su salud física o los problemas emocionales han dificultado sus actividades sociales habituales con la familia, los amigos, los vecinos y otras personas? (marque un solo número)

Nada...............................1

Un poco.............................2

Regular.............................3

Bastante.............................4

Mucho...............................5

¿tuvo dolor en alguna parte del cuerpo durante las 4 últimas semanas? (marque un solo número)

No, ninguno.........................1

Sí, muy poco........................2

$\mathrm{Si}$, un poco.......................... 3

$\mathrm{Si}$, moderado.......................4

Sí, mucho.............................5

Si, muchísimo........................6

Durante las 4 últimas semanas, ¿hasta qué punto el dolor le ha dificultado su trabajo habitual (incluido el trabajo fuera de casa y las tareas domésticas)? (marque un solo número)

Nada. 1

Un poco............................2

Regular..............................

Bastante.............................4

Mucho.................................

Las preguntas que siguen se refieren a cómo se ha sentido y como le han ido las cosas durante las 4 últimas semanas. En cada pregunta responda lo que se parezca más a como se ha sentido usted. Durante las últimas 4 semanas ¿Cuánto tiempo...(marque un solo número por cada pregunta)

\begin{tabular}{|l|c|c|c|c|c|c|}
\hline & Siempre & Casi siempre & Muchas veces & Algunas veces & Sólo alguna vez & Nunca \\
\hline $\begin{array}{l}\text { a. ¿Se sintió lleno de } \\
\text { vitalidad? }\end{array}$ & $\mathbf{1}$ & $\mathbf{2}$ & $\mathbf{3}$ & $\mathbf{4}$ & $\mathbf{5}$ & $\mathbf{6}$ \\
\hline $\begin{array}{l}\text { b. ¿estuvo muy nervioso? } \\
\mathbf{1}\end{array}$ & $\mathbf{2}$ & $\mathbf{3}$ & $\mathbf{4}$ & $\mathbf{5}$ & $\mathbf{6}$ \\
\hline $\begin{array}{l}\text { c. ¿se sintió tan baja de moral } \\
\text { que nada le permitía realizar? } \\
\text { Podía animarle? }\end{array}$ & $\mathbf{1}$ & $\mathbf{2}$ & $\mathbf{3}$ & $\mathbf{4}$ & $\mathbf{5}$ & $\mathbf{6}$ \\
\hline $\begin{array}{l}\text { d. Se sintió calmado y } \\
\text { tranquilo? }\end{array}$ & $\mathbf{1}$ & $\mathbf{2}$ & $\mathbf{3}$ & $\mathbf{4}$ & $\mathbf{5}$ & $\mathbf{6}$ \\
\hline e. tuvo mucha energía? & $\mathbf{1}$ & $\mathbf{2}$ & $\mathbf{3}$ & $\mathbf{4}$ & $\mathbf{5}$ & $\mathbf{6}$ \\
\hline g. Se sintió agotado? & $\mathbf{1}$ & $\mathbf{2}$ & $\mathbf{3}$ & $\mathbf{4}$ & $\mathbf{5}$ & $\mathbf{6}$ \\
\hline
\end{tabular}




\begin{tabular}{|l|l|l|l|l|l|l|}
\hline h. Se sintió feliz? & $\mathbf{1}$ & $\mathbf{2}$ & $\mathbf{3}$ & $\mathbf{4}$ & $\mathbf{5}$ & $\mathbf{6}$ \\
\hline i. Se sintió cansado? & $\mathbf{1}$ & $\mathbf{2}$ & $\mathbf{3}$ & $\mathbf{4}$ & $\mathbf{5}$ & $\mathbf{6}$ \\
\hline $\begin{array}{l}\text { f. Se sintió desanimado y } \\
\text { triste? }\end{array}$ & $\mathbf{1}$ & $\mathbf{2}$ & $\mathbf{3}$ & $\mathbf{4}$ & $\mathbf{5}$ & $\mathbf{6}$ \\
\hline
\end{tabular}

Durante las 4 últimas semanas, ¿con qué frecuencia la salud física o los problemas emocionales le han dificultado sus actividades sociales (como visitar a los amigos o familiares)? (marque un solo número)

Siempre. $\ldots 1$

Casi siempre. .2

Algunas veces. .3

Sólo alguna vez...........................4

Nunca.................................................

Por favor, diga si le parece CIERTA o FALSA cada una de las siguientes frases: (marque un solo número por cada pregunta)

\begin{tabular}{|l|c|c|c|c|}
\hline & $\begin{array}{c}\text { Totalmente } \\
\text { cierta }\end{array}$ & $\begin{array}{c}\text { Bastante } \\
\text { cierta }\end{array}$ & $\begin{array}{c}\text { Bastante } \\
\text { falsa }\end{array}$ & $\begin{array}{c}\text { Totalmente } \\
\text { falsa }\end{array}$ \\
\hline a. Creo que me pongo enfermo más fácilmente que otras & $\mathbf{1}$ & $\mathbf{2}$ & $\mathbf{3}$ & $\mathbf{4}$ \\
\hline b. Estoy tan sano como cualquiera. & $\mathbf{1}$ & $\mathbf{2}$ & $\mathbf{3}$ & $\mathbf{4}$ \\
\hline c. Creo que mi salud va a empeorar & $\mathbf{1}$ & $\mathbf{2}$ & $\mathbf{3}$ & $\mathbf{4}$ \\
\hline d. Mi salud es excelente & $\mathbf{1}$ & $\mathbf{2}$ & $\mathbf{3}$ & $\mathbf{4}$ \\
\hline
\end{tabular}

¡GRACIAS POR SU COLABORACIÓN! 